\title{
La formación de profesores de ciencias a través de su interacción en Comunidades de Desarrollo Profesional
}

\author{
Álvaro García Martínez* \\ Artículo recibido: 03-09-2009 y aprobado: 12-11-2009 \\ The training of science teachers through its interaction \\ Professional Development Communities
}

\begin{abstract}
Resumen: La formación del profesorado se ha venido trabajando desde diferentes perspectivas, en este documento se plantea una propuesta para abordarla desde de la creación de comunidades que tienen como objeto el desarrollo profesional de sus participantes. El modelo se desarrolla en cinco fases que intentan modificar los ámbitos de formación de los profesores para que poco a poco vayan actuando como diseñadores críticos y reflexivos. Este modelo se fundamenta en el uso del diseño curricular como una vía imprescindible para lograr el desarrollo profesional de los profesores durante su interacción con la comunidad. Como caso particular se presenta una experiencia de implementación del proceso de formación adelantada con profesores universitarios quienes trabajan en el área de química.
\end{abstract}

Palabras clave: Comunidades de desarrollo profesional, formación profesional, diseño curricular.
Abstract: Traditional teacher training has been worked since many perspectives, in this paper we pretend to broach it since the consolidation of communities which object is the professional development of their members. The model is developed in five phases, where each one of them tries to modify the fields of the teachers' education becoming them into critic and thoughtful designers. This model is supported on the use of the curricular designing as the core to achieve the professional development of teachers during their interaction with the community. As a particular case, we show the implementation of the process followed by professors who work at the chemistry area.

Key words: Communities professional development, training, curriculum design.

Profesor del proyecto Curricular de Licenciatura en Química. Facultad de Ciencias y Educación. Universidad Distrital Francisco José de Caldas. alvaro.garcia@udistrital.edu.co 


\section{Introducción}

La formación del profesorado ha ocupado un espacio de gran importancia en las investigaciones que se adelantan en Didáctica de las Ciencias, se ha abordado tanto en el nivel inicial como en el ejercicio y en diferentes niveles de formación, profesores de primaria, secundaria y universitarios. Se han desarrollado estudios sobre diferentes aspectos de la formación docente, sus ideas y creencias desde la naturaleza de la ciencia, sobre la enseñanza, sobre el aprendizaje y prácticas docentes, entre otras.

También se ha estudiado la influencia de las emociones, la identidad y lo humano del trabajo docente en su desempeño profesional (O'Connor, 2008), los cuales son contemplados desde estándares profesionales para evaluar el desempeño del profesor en algunos países como Australia. O'Connor desarrolla un estudio en donde pone de manifiesto la gran influencia que tienen las emociones y el carácter humano en el ejercicio docente y como éstos elementos se evidencian en dimensiones de tipo profesional, de desempeño y filosófico, e insiste en la importancia de tenerlas en cuenta a la hora de hablar de "Estándares Profesionales para la Enseñanza".

Ciertos proyectos de desarrollo profesional tienen en común el apoyo que brindan a los profesores para examinar y cambiar tanto sus creencias y su conocimiento como su práctica docente. Consideran lo suficientemente importantes las creencias y el conocimiento que poseen los profesores como para someterlos a un examen detenido y a una posible transformación. De otro lado, ponen de relieve la enseñanza de una materia docente concreta en lugar de centrarse en enfoques generalistas o universales sobre la enseñanza. Lo relevante es que, con un trabajo sistemático e intenso, los profesores lleven a cabo cambios sustanciales en su conocimiento, sus creencias y sus prácticas, cambios que tienen unas orientaciones compatibles con una visión que apuesta por la reforma de la enseñanza y del aprendizaje (Putnam y Borko, 1997).

A pesar de los estudios realizados, cuando se habla de la formación del profesor de ciencias que se encuentra en ejercicio, surgen interrogantes como los siguientes: ¿cuáles serían los fines de dicha formación, cómo debe realizarse, qué se debe trabajar con los docentes, debe centrarse en una formación en el cómo enseñar o incluir elementos de la disciplina misma? En fin, realizar toda una planeación curricular para la estructuración de un programa de formación y actualización docente.

Analizando la coherencia de los objetivos formativos con las demandas que día a día se le presentan al profesorado para que llegue a ser considerado como un profesional experto, se pueden plantear cuatro grandes metas, en cuanto a los programas de formación se refiere (Valcacer y Sánchez, 2000):

- Mejorar el conocimiento de los profesores en relación con la asignatura que enseñan, sobre todo mediante un aumento del conocimiento del contenido de enseñanza y del conocimiento didáctico del contenido.

- Cambiar las concepciones y prácticas docentes de los profesores hacia enfoques coherentes con presupuestos constructivistas.

- Formar al profesor como diseñador de proyectos curriculares e investigador de su actuación docente en el aula. 
- Desarrollar actitudes y prácticas docentes más colaborativas, críticas y autónomas.

Alrededor de estas metas se han adelantado diversidad de trabajos de investigación que intentan generar mejores propuestas sobre cómo formar a los profesores de ciencias, para nuestro caso, nos hemos orientado por la creación de comunidades que trabajan en el diseño curricular para potenciar su desarrollo profesional docente.

\section{Desarrollo}

El área de formación del profesorado ha evolucionado en la formulación de orientaciones teóricas y metodológicas, por lo cual la perspectiva en los programas ha venido cambiando y con ella, la terminología, expresiones como Teacher Education o Teacher Training van desapareciendo debido a las implicaciones que acarrea su uso y aunque algunos autores aún las emplean, para evitar ser ubicados en la clásica imagen reduccionista, hacen las respectivas aclaraciones y especifican lo que entienden por éstos, últimamente se emplea el término desarrollo del profesor o desarrollo profesional del profesorado (Teacher Development o Teacher Professional Development).

No hay que plantear la formación del profesorado como un "cambio", sino más bien como un proceso interno de "crecimiento" y "desarrollo" gradual que parte de lo que ya piensan y hacen, de los problemas reales de enseñanza y aprendizaje de las ciencias, de las preocupaciones cotidianas del profesor, potenciando y apoyando la motivación, la disponibilidad, la colaboración y el compromiso de los profesores en su propio desarrollo profesional (Mellado, 2003).

Nosotros asumimos el desarrollo profesional del profesorado como una serie de procesos sucesivos de autorregulación metacognitiva que llevan a un crecimiento en los ámbitos que orientan la profesión docente (ámbito personal, ámbito práctico y de conocimientos y estructuras interpretativas, todos ellos en el mundo personal del profesor) producto de la comprensión, puesta en práctica y de la relación entre lo que piensan, sienten y hacen en su aula y en su institución; dichos ámbitos se ven influenciados permanentemente por el ámbito externo (García, 2009).

Este desarrollo profesional implica que los profesores y profesoras asuman el hecho de que pueden aprender desde la diversidad, desde lo social, desde el contexto, desde la actividad intelectual compartida, siendo conscientes además de su proceso de aprendizaje personal. Cochran-Smith (1998) plantea que el desarrollo profesional de los profesores es "una construcción de cultura" no un entrenamiento de habilidades; investigaciones sobre el trabajo de los profesores y sus sitios de trabajo sugieren que las mejores prácticas de formación del profesorado son aquellas que les proporcionan oportunidades para identificar, reconsiderar, soportar o modificar creencias sobre el salón de clase, además de las prácticas que soporten o profundicen en las oportunidades de aprendizaje de los estudiantes y en oportunidades para la vida.

Trabajar desde una perspectiva colaborativa e interdisciplinaria ha sido probablemente uno de los retos más grandes; no es fácil generar un clima 
colaborativo dentro de culturas cuyas características típicas son el trabajo individualizado y anquilosado que se desarrolla en la universidad. Es difícil crear un escenario común en el que los profesores sean capaces de trabajar juntos si persisten las diferencias en sus puntos de vista sobre la enseñanza. Así, un primer paso para estudiar las prácticas de enseñanza en la universidad es dejar de lado el estudio de los contenidos y centrarse más en el trabajo en equipos y en las reflexiones sobre la práctica.

Para que los profesores puedan desenvolverse de forma óptima en estos contextos se requerirán, en muchos casos, importantes cambios en su conocimiento, sus creencias y sus prácticas. Esto implica concebir propuestas de formación del profesorado que lo oriente hacia una actuación más acorde con los retos de este nuevo milenio, lo cual involucra plantear programas de formación que se basen en experiencias exitosas en formación del profesorado y que tengan presentes elementos como los mencionados por Putnam y Borko (1997):

- Los profesores deberían ser tratados como personas que están aprendiendo activamente y que construyen sus propias interpretaciones.

- Debería reconocerse el poder de los profesores y deberían ser tratados como profesionales.

- La formación del profesorado debe centrarse en la práctica del aula.

- Los profesores que forman al profesorado deberían tratar a los maestros de la misma manera en que esperan que los maestros traten a sus alumnos.
Putnam y Borko (1997) han denominado a estos tópicos como mantras, ya que quieren reconocer no solo la importancia de las ideas que subyacen a estas afirmaciones sino la precaución que hay que tener para no simplificarlas en demasía y hacer de ellas un uso indiscriminado.

En cuanto al término comunidades de aprendizaje, utilizado cada vez con mayor frecuencia, involucra expresiones como comunidades de trabajo y comunidades colaborativas, llegando al punto de referirse a una comunidad virtual, en la que se puede pagar por un servicio para tener acceso a él, en ésta se le asigna un nombre de usuario y una clave de acceso, imágenes tradicionales de las que nos alejamos en lo que hemos desarrollado.

Por otra parte es posible contar con tesis como la de Resnik (1991) quien habla de comunidades de discurso, aquellas comunidades que comparten formas de pensamiento y comunicación a través de la interacción continua, es decir que los individuos han llegado a compartir formas comunes de pensar y expresar sus ideas. En esta línea de pensamiento surge el aprendizaje en comunidades, el cual se interpreta como aquel conjunto amplio y heterogéneo de metodologías de enseñanza, organizadas y estructuradas, que orientan las actividades de los individuos cuando trabajan juntos, en grupo o en equipos, en situaciones que los convocan para lograr un propósito determinado.

Es posible encontrar al momento de buscar literatura acerca de comunidades de profesionales, cierta cantidad de títulos sobre la temática, pero al indagar acerca de comunidades de profesores los resultados son mucho menores, esto 
significa que hay mucho por hacer. Se han estudiado comunidades de otras profesiones como medicina o leyes, pero al reflexionar un poco sobre una comunidad de profesores se observa que a diferencia de otras, es muy poco lo que el profesor puede decidir sobre su propia comunidad, ya que la mayoría de lo que él trabaja cotidianamente se hace por mandatos de ámbitos superiores como los constructores de políticas educativas y de personas que están lejos de la realidad de las aulas de clase de hoy. Comparada con medicina o las leyes, la educación no tiene la posibilidad de crear un lenguaje compartido de normas y valores.

En el trabajo de comunidades de profesores es necesario destacar su carácter local, donde las interacciones cara a cara, el diálogo constructivo y la confianza, son elementos indispensables para la construcción de la cohesión en el grupo. Una definición interesante de comunidad es la que presentan Bellah et al. (1985), "un grupo de personas que son socialmente interdependientes, quienes participan entre sí en discusiones y tomando decisiones, y quienes comparten ciertas prácticas que definen la comunidad y son nutridas por ésta”. De esta definición se puede concluir que este es un proceso lento y pensado a largo plazo, ya que los individuos en sus diálogos e interacciones van construyendo una historia como grupo, lo cual les va dando identidad al ir compartiendo cada vez más elementos y construyendo propuestas a partir del trabajo en equipo.

Un aspecto clave de las comunidades de profesores es que crean continuamente espacios de aprendizaje de los profesores (Cochran-Smith, 1998). Sin embargo, la gran cantidad de asignación de cursos, en el caso de educación media, el número de estudiantes en los cursos y el total por día son muy altos, hechos que impiden la conformación de espacios para que los profesores puedan trabajar en equipo, teniendo como consecuencia que el único espacio disponible para ello sea el del descanso escolar en una jornada de trabajo que alcanza solo para comer algo. El caso de la educación superior varía un poco, ya que al hacer este proceso como algo formal, se pueden generar los intervalos de tiempo necesarios.

Las discusiones sobre la práctica son de gran importancia en la medida en que los profesores pueden hablar sobre casos concretos y presentar diferentes puntos de vista sobre los mismos, generando un ambiente de reflexión crítica permanente de tipo metacognitivo. Cuando se participa en estos seminarios, los profesores están aprendiendo nuevas formas de aprendizaje sobre su práctica docente y al mismo tiempo están construyendo nuevas formas de discurso para hablar de su trabajo docente.

Tomando como referencia lo mencionado, interpretamos a la comunidad de desarrollo profesional de profesores, CODEP, como:

Un grupo de profesores que se reconocen como profesionales de la educación, los cuales participan en discusiones críticas y reflexivas para la toma de decisiones en torno a su objeto de estudio, la enseñanza y el aprendizaje en su aula (e institución) y otros procesos que allí se desarrollan y que las condicionan. Esta comunidad comparte poco a poco ciertas prácticas y referentes que se van construyendo como producto 
de esta dinámica, que la definen y retroalimentan.

Cuando se dice que los profesores deben reconocerse como profesionales, se hace referencia al reconocimiento que ellos mismos crean como profesores universitarios, por ejemplo si su objeto de estudio es la enseñanza de la química (que va de la mano con el cómo aprenden sus estudiantes) éste se acompaña de todas las implicaciones que el ejercicio conlleva en su aula y su institución; esta técnica se fue logrando poco a poco y en la medida en que se consolidó la comunidad.

La comunidad de desarrollo profesional de profesores que se describe a continuación, se diseñó a partir de los referentes descritos y se implementó con profesores universitarios del área de química durante dos años'. Se tomó como eje de estudio y reflexión, con los profesores universitarios, el uso de la historia de la química para el mejoramiento de la docencia universitaria en los casos particulares de la química general y la fisicoquímica. La CODEP se ha soportado en referentes particulares de los programas de su clase, pero al mismo tiempo presenta elementos propios que la diferencian de otras propuestas, los cuales se describen a continuación:

Lo primero que se trabajó con los profesores, cuando se realizó el programa de formación, fue el cómo enseñar unos contenidos estudiados y reflexionados a la luz de la historia de la química, estos contenidos fueron analizados y construidos bajo los principios

1 Los profesores universitarios tienen título de químicos e ingenieros químicos, con maestrías y doctorado en química. de comunidad de aprendizaje a nivel profesional; desde aquí surge el segundo aspecto, contribuir a la formación de un profesional que vea la necesidad de trabajar en equipo, de socializar sus conocimientos, sus aprendizajes y obviamente, sus problemas, a la hora de entrar en acción. Finalmente, estos elementos contribuyeron a la formación de una perspectiva que busca el desarrollo del profesor como un profesional que se interroga y formula problemas de investigación sobre su práctica docente, que diseña y lleva al aula propuestas de innovación buscando superar problemas y poner a prueba hipótesis de trabajo consensuadas en equipo, construyendo y reconstruyendo nuevamente conocimiento didáctico de su disciplina de formación básica.

Los profesores universitarios se vincularon al programa de manera voluntaria y con la intención de mejorar su docencia. El carácter voluntario es un elemento fundamental que permite un alto grado de pertenencia con la comunidad, sin embargo no implica que todos tengan la misma responsabilidad con la comunidad ni con sus actividades, pero ayuda muchísimo para el desarrollo de las tareas propuestas y el logro de la metas fijadas.

El coordinador de la CODEP, y autor de este documento, tuvo que desarrollar distintos roles, entre ellos, el de negociador de intereses, haciendo por un lado, que los objetivos de la investigación no se diluyeran y, al mismo tiempo, responder a las expectativas de los miembros de la comunidad. Un segundo rol fue el de líder académico, generando confianza en el grupo de tal forma que las actividades propuestas no fuesen vistas como 
una "pérdida de tiempo" que no tendrían continuidad; un tercer rol fue su desempeño como persona, capaz de escuchary valorar lo que los otros miembros dicen $\mathrm{y}$ hacen, que al tiempo, los motivaba y presionaba cuando era necesario, pero lo más importante fue su capacidad para transmitir a los asistentes la idea de que cumplían un papel protagónico en la tarea de ser constructores de conocimiento didáctico, siendo con ello un compañero que aporta, cuestiona y presenta alternativas.

Con lo anterior se quiere plantear que la persona que orienta el proceso de formación del profesorado debe tener ciertas características que le permitan generar un ambiente propicio para el desarrollo de las actividades previstas; así, Richardson (1992) afirma que el responsable del desarrollo del profesorado en un contexto de este tipo debe tener un sólido conocimiento sobre la investigación, porque la presentación de este conocimiento es más útil cuando surge como respuesta a las cuestiones suscitadas en el curso de la conversación. El responsable del desarrollo del profesorado también ha de tener cuidado en ser humilde, incluso modesto, al presentar la información y los puntos de vista, para así enfrentar con mayor propiedad la idea, dominante en las escuelas, de que los responsables del desarrollo del profesorado vinculados a la universidad son los "expertos".

La CODEP fue planteada a los profesores como un intento por contribuir al mejoramiento de la docencia universitaria de los participantes, su orientación en este caso particular ha sido tener en cuenta las relaciones entre la historia de la química, la filosofía de las ciencias y la didáctica de la química, como elementos que fundamentan las acciones docentes. Una vez ellos estuvieron de acuerdo con el objetivo, se organizó un plan de trabajo en conjunto que les dio la posibilidad de desarrollar sus propias ideas y aportar desde su experiencia personal sin descuidar el objetivo del proceso que se había previsto, es decir, los contenidos básicos del programa.

Los aspectos fundamentales para llevar a cabo la agenda de una comunidad son el tiempo y el espacio, y fueron alcanzados gracias a que se logró una descarga académica de dos horas a la semana para los profesores participantes del proyecto y se adaptó al horario de cada uno de ellos para que tuvieran un día a la semana para trabajar en la comunidad; este tiempo se prolongó en algunos casos, ya que ellos fueron moviendo sus actividades antes o después de la franja, dependiendo de las necesidades y de sus posibilidades de horario y de trabajo.

En cuanto al espacio, las actividades se desarrollaron en la oficina de un grupo de investigación dirigido por el autor y se contó con los laboratorios de química para realizar los trabajos experimentales necesarios. En este sentido, Grossman y Wineburg (2000) manifiestan que es indispensable, al hablar de una comunidad profesional, que el lugar de reuniones sea la misma institución y que se desarrolle en la jornada de trabajo de los profesores, ofreciendo la posibilidad de generar una transformación individual al tiempo que una transformación en la organización social del espacio en el que los individuos trabajan. Estos aspectos son complejos para los casos de primaria y secundaria por la cantidad de horas 
semanales de trabajo que se tienen y el número de estudiantes.

Para el desarrollo de la CODEP se han programado cinco fases:

Fase 1. Identificación de propósitos y selección de contenidos.

Fase 2. Selección del tópico y área de la historia de la ciencia a estudiar.

Fase 3. Diseño de la herramienta de enseñanza/aprendizaje.

Fase 4. Implementación y análisis del proceso de aplicación de la herramienta.

Fase 5. Reflexión metacognitiva sobre el proceso desarrollado.

En la primera fase de la CODEP se pretende, en primera instancia, identificar las ideas del profesorado sobre tópicos que se consideran de relevancia para el desarrollo del programa, los cuales pueden ser aspectos que condicionen, proyecten y sean fundamentales para el desarrollo profesional del profesorado.

Retomando el diagnóstico anterior y continuando con el trabajo de comunidades, se inicia la segunda fase, en ella se determina el tópico de estudio que se podría y sería pertinente abordar. De esta manera se selecciona el tipo de orientación más interesante y pertinente, desde la historia de las ciencias, para trabajar con los profesores, entre las opciones que se pueden tomar se cuenta con: ciencia y cultura, comunicación en ciencia, género, instrumentos y prácticas experimentales, cuadernos de notas, etc.

En la tercera fase se pretende evidenciar la manera como interpretan los profesores el uso de la historia de las ciencias en un contexto escolar y qué análisis realizan para su posterior im- plementación. De igual manera permite reflexionar sobre como ellos conciben el mejoramiento de su enseñanza, promoviendo el desarrollo de aprendizajes significativos mediante el empleo de la historia de la ciencia.

En la cuarta fase se contrastan los criterios que planteó el profesor para el diseño de la unidad didáctica y la manera como los retoma al momento de su implementación. Se presta especial interés en la forma en que desarrolla cada una de las actividades y los elementos que resalta, sean estos positivos o negativos, con el fin de comprobar la relación de sus ideas y como éstas justifican su propuesta.

En la última fase se analizan los posibles cambios que se presentaron en los profesores que conforman la CODEP, bien en sus actitudes, su lenguaje o sus acciones. Se realiza de igual forma una reflexión metacognitiva sobre las relaciones historia, filosofía y enseñanza de las ciencias.

\section{Conclusiones}

El trabajo en comunidades de desarrollo profesional ubica a los docentes en un papel de aprendices, hecho que facilita la construcción de propuestas a partir del trabajo colectivo, posibilitando la generación de actitudes críticas que a su vez permiten la creación de reflexiones metacognitivas de forma permanente en los participantes.

Las prácticas docentes de los profesores fueron cambiando progresivamente en la medida en que se fueron apropiando de su papel como diseñadores de sus propias asignaturas, esto se da debido al trabajo en la comunidad con los otros profesores. Se rediseñaron 
los cursos completos de cada asignatura a la luz de perspectivas constructivistas de resolución de problemas soportadas en el uso de la historia de la química.

El trabajar en propuestas que impliquen diseño curricular posibilita el establecimiento de todo un proceso de

\section{Bibliografía}

Bellah, R. N., Madsen, N., Sullivan, W. M., Swidler, A., \& Tipton, S. M. (1985). Habits of the heart; individualism and commitment in american life. Berkeley, CA: University of California Press.

Cochran-Smith, M. (1998). Teaching for social change: toward a grounded theory of teacher education. En A. Hargreaves., A. Lieberman., M. Fullan., \& D. Hopkins. (Eds.). The International Handbook of Educational Change. Ámsterdam: Kluwer Academic.

García, M. (2009). Aportes de la historia de la ciencia al desarrollo profesional de profesores de química. Tesis Doctoral no publicada. Universidad Autónoma de Barcelona.

Grossman, P. y Wineburg, S. (2000). ¿What makes teacher community different from a gathering of teachers? Centre for the study of teaching and policy. Washington: University of Washington. desarrollo profesional de los profesores, en la medida en que influye de manera directa en los ámbitos de la profesión docente, lo cual se observó en los participantes al analizar sus ideas, acciones y los productos generados.

Mellado, V. (2003). Cambio didáctico del profesorado de ciencias experimentales y filosofía de la ciencia. Enseñanza de las ciencias. 21 (3).

O'Connor, Kate. (2008). "You choose to care": teachers, emotions and professional identity. Teaching and Teacher Education. Vol. 24.

Putnam, R. T. \& Borko, H. (1997). Teacher learning: implications of new views of cognition. En B. J.

Biddle, et al. (Eds.). International Handbook of Teachers and Teaching. Ámsterdam: Kluwer Academic.

Resnick. (1991). Shared cognition: thinking as social practice. En Resnick, Levine y Teasley (Comps.). Perspectives on socially shared cognition. Washington, DC: American Psychological Association.

Valcárcel y Sánchez (2000). La formación del profesorado en ejercicio. En Perales y Cañal. Didáctica de las ciencias experimentales. Marfil. 